\title{
Shouting to be Heard in Advertising
}

\author{
Simon P. Anderson and André de Palma*
}

July 2011 revised October 2012

\begin{abstract}
Advertising competes for scarce consumer attention, so more profitable advertisers send more messages to break through the others' clutter. Multiple equilibria can arise: more messages in aggregate induce more "shouting to be heard", dissipating profit. Equilibria can involve a small range of loud shouters or large range of quiet whisperers. All advertisers prefer there to be less shouting. There is the largest diversity in message levels for a middling width of advertiser types: both a very wide or very narrow width have only one message per advertiser. The number of advertisers at each message level decreases with the level if the profit distribution is log-convex. Increasing the cost of sending messages can make all advertisers better off. A new technique is given for describing multiple equilibria, by determining how much examination is consistent with a given marginal advertiser.
\end{abstract}

Jel Classification: D11, D60, L13.

KEYWORDS: information overload, congestion, advertising, lottery, junk mail, e-mail, telemarketing, multiple equilibria, ad caps.

${ }^{*}$ Simon P. Anderson: Department of Economics, University of Virginia, PO Box 400182, Charlottesville VA 22904-4128, USA, sa9w@virginia.edu. André de Palma: ENS-Cachan (Economics and Management Department), Centre d'Economie de la Sorbonne, FRANCE. andre.depalma@ens-cachan.fr. The first author gratefully acknowledges research funding from the NSF under grant SES-0452864 and from the Bankard Fund at the University of Virginia. We thank various seminar participants and conference participants at SWIO 2006 (Auckland, NZ), CRETE (Rethmynon, 2006), IIOC 2007 (Savannah, GA), CEPR 2007 (Taragonna), the Workshop on the Psychology and Economics of Attention (Toulouse, 2011), and the Paris School of Economics (2012), as well as Jim Dana, Martin Peitz, and Sanjay Raneev (Cambridge), two referees and an Associate Editor for perceptive comments, and Charlie Murry for research assistance. 
Well, it's a non-stop blitz of advertising messages. Everywhere we turn we're saturated with advertising messages trying to get our attention. We've gone from being exposed to about 500 ads a day back in the 1970 's to as many as 5,000 a day today. We have to screen it out because we simply can't absorb that much information. We can't process that much data and so no surprise, consumers are reacting negatively to the kind of marketing blitz; the kind of super saturation of advertising that they're exposed to on a daily basis. All of this marketing saturation that's going on is creating this kind of arms race between marketers where they have to up the ante the next time out because their competitors have upped the ante the last time they were out. And the only way you can win is to have more saturation. ${ }^{1}$

\section{Introduction}

The volume of conversation at a cocktail party is low when people are talking normally, but high when everyone has to shout to be heard over the din caused by the others trying to do the same thing. A parallel can be drawn with the volume of advertising messages trying to get through to consumers: firms must send many ads to break through into the consumer's consideration set (see Terui, Ban, and Allenby, 2011, for recent work on consideration sets in marketing.) Consumers can scarcely process all the incoming information, and typically disregard a lot of the messages projected at them. In such a world, advertisers are more likely to connect if they send several messages: advertisers escalate to break through the advertising clutter of others' messages in order to attract attention and eventually get to a sale. This paper is about duplication and repetition of advertising messages, information overload, the possibility of multiple equilibria with higher volume of messages sent by fewer advertisers, and potential gains from raising the cost of sending messages.

The economics literature on advertising is a vast one and is reviewed in Bagwell (2007). Butters' (1977) model of informative advertising and price dispersion assumes that firms within the same industry send messages announcing the (availability and) price of a good. Messages are sent randomly to consumers, so each consumer will get a different profile of messages. Each firm sends a single message, which is a zero-profit activity under the assumption of free-entry of senders. In Grossman and Shapiro (1984), firms form an oligopoly (also within the same industry), and each firm sends multiple messages, again randomly distributed over receivers, in order to reach a desired

\footnotetext{
${ }^{1}$ President Jay Walker-Smith of the Marketing Firm Yankelovich (Excerpted from Cutting Through Advertising Clutter, CBS News.) http://www.cbsnews.com/stories/2006/09/17/sunday/main2015684.shtml
} 
customer base. All messages are assumed to be read by the recipient, and firms are symmetric.

By contrast, we look at heterogenous advertisers from different industries interacting only through a bottleneck of limited individual attention span, with advertisers sending multiple messages even though they know to whom they are sending their messages. ${ }^{2}$ In trying to overcome congestion by duplication, advertisers exacerbate it. When firms can send multiple messages, there may be overcrowding in both depth (messages per advertiser) and width (number of advertisers). The depth dimension provides a further perspective on consumer attention overload problem analyzed by Van Zandt (2004), and by Anderson and de Palma (2009). These authors assumed that each advertiser can send at most one message (i.e., no depth choice).

We show that multiple equilibria can exist: when there is less advertiser width, there is much greater depth for high-volume advertisers and so more message volume in total. This underlies the property of "shouting to be heard": fewer advertisers shout but at much higher volume.

Increasing the cost of sending each message might be expected to help in two dimensions. First, one might expect there to be fewer advertiser types (the low end drops off) and less competition (fewer messages sent) at any given level. Instead, however, we show it is possible that raising the cost of sending messages causes more advertisers to send, and fewer messages in total. A cost increase actually makes all advertisers better off. This result emphasizes the Prisoners' Dilemma feature of the equilibrium. Even highly profitable advertiser types that send a lot of messages see their profits rise through an increase in the probability they are examined. The high types now are more "prominent" in that their messages get through more clearly (less clutter from others), and this raises aggregate profits from advertising.

\section{An Example}

Consider two firms, and one consumer. The consumer will examine only two messages. It costs a firm $\gamma$ to send a message, and its profit is $\pi>\gamma$ if its message is examined. There is an equilibrium with each firm sending a single message, in which case firms each earn $\pi-\gamma$.

\footnotetext{
${ }^{2}$ Extending our model to allow for uncertainty about whether the recipient got the message (which is the reason for sending multiple messages in Grossman and Shapiro, 1984) ought not greatly alter our conclusions.
} 
There is also an equilibrium with the firms (A and B) sending two messages each if $\pi>12 \gamma$, and the firms are constrained to send no more than two messages. To see this, it suffices to write out the pay-off matrix for the game: ${ }^{3}$

\begin{tabular}{|c|l|c|}
\hline$A \backslash B$ & 1 & 2 \\
\hline 1 & $\pi-\gamma, \pi-\gamma$ & $\frac{2 \pi}{3}-\gamma, \pi-2 \gamma$ \\
\hline 2 & $\pi-2 \gamma, \frac{2 \pi}{3}-\gamma$ & $\frac{3 \pi}{4}-2 \gamma, \frac{3 \pi}{4}-2 \gamma$ \\
\hline
\end{tabular}

There are thus multiple equilibria. When both firms send two messages, there is Shouting to be Heard. This equilibrium is worse for both firms than when each sends one message, and it is worse for the consumer insofar as it may entail only one firm's messages being examined.

Now drop the restriction that at most two messages can be sent (but the number of messages examined, $\phi$ is still two). To find the symmetric pure strategy equilibria, it suffices (by concavity of profits in number of messages sent) to find the pay-offs for an advertiser deviating to one more or one less message than its rival. Consider the case when both send $k$ messages. The chance that $B$ 's first message is examined is $\frac{1}{2}$, and the chance that the first is missed is also $\frac{1}{2}$. Given the first misses, the chance the second is examined is $\frac{k}{2 k-1}$. Summing, the chance of being examined when both send $k$ messages is $\frac{1}{2}\left(\frac{3 k-1}{2 k-1}\right)$. Analogous calculations give the pay-offs to advertiser $B$ in the following table (for $k>1):^{4}$

\begin{tabular}{|l|l|l|l|l|l|}
\hline$A \backslash B$ & $\ldots$ & $k-1$ & $k$ & $k+1$ & $\ldots$ \\
\hline$\ldots$ & & & & & \\
\hline$k$ & & $\frac{3 k-2}{2(2 k-1)} \pi-(k-1) \gamma$ & $\frac{1}{2} \frac{3 k-1}{2 k-1} \pi-k \gamma$ & $\frac{3(k+1)}{2(2 k+1)} \pi-(k+1) \gamma$ & \\
\hline$\ldots$ & & & & & \\
\hline
\end{tabular}

From these pay-offs, we can determine the conditions for $(k, k)$ to be an equilibrium: this is when the middle term exceeds the two neighboring ones (i.e., $B$ does not wish to deviate to one more or one less message). Hence, $(k, k)$ is an equilibrium if

$$
\frac{\pi}{\gamma} \in\left[2(2 k-1), \frac{(2 k+1)(2 k-1)}{(k-1)}\right], \quad k=2,3, \ldots
$$

\footnotetext{
${ }^{3}$ There is also a symmetric mixed strategy equilibrium at which the probability each firm sends one message is $\mathbb{P}_{1}=\left(1-12 \frac{\gamma}{\pi}\right)$, and the probability of sending two messages is the complementary probability.

${ }^{4}$ For $k=1$ the payoff when $B$ chooses $0(k-1)$ messages is 0 .
} 
As noted above, $(1,1)$ is always an equilibrium for $\frac{\pi}{\gamma} \geq 1$. From $(1),(2,2)$ is an equilibrium for $\frac{\pi}{\gamma} \in[6,15] ;(3,3)$ for $\frac{\pi}{\gamma} \in\left[10, \frac{35}{2}\right] ;(4,4)$ for $\frac{\pi}{\gamma} \in[14,21] ;(5,5)$ for $\frac{\pi}{\gamma} \in\left[18, \frac{99}{4}\right]$; and $(6,6)$ for $\frac{\pi}{\gamma} \in\left[22, \frac{143}{5}\right]$. Hence there are 4 symmetric equilibria (with advertisers choosing each $1,2,3$, or 4 messages) for $\frac{\pi}{\gamma}=14$. For $\frac{\pi}{\gamma}=20$, there are equilibria with 1 , 4 , and 5 messages each, so there is a "hole" in the equilibrium set. These examples show that there are multiple equilibria. The ones with more messages sent (more shouting) are worse for advertisers (higher costs and lower chance of a sale through more duplication).

We can illustrate the effects of lower consumer attention span by looking at $\phi=1$. Consider again the case where at most 2 messages can be sent per advertiser. It is readily shown that pure strategy equilibrium is almost always unique (up to permuting advertisers). ${ }^{5}$ If $\frac{\pi}{\gamma} \in[6,12]$, firms send 2 messages each, while they send only 1 message each for $\phi=2$. Hence a lower examination rate engenders more shouting.

\section{Model}

The model describes a continuum of senders of messages (advertisers) facing a potential consumer whose attention is restricted to only process a limited number of messages (see Eppler and Mengis, 2004, for a review of the interdisciplinary literature on information overload, and the early work by Miller, 1956). Sending more messages is more costly for a sender, but increases the probability of breaking through. With a fixed consumer attention span, sending messages is like a lottery where there are multiple winners. Participants typically value a winning connection differently because they enjoy different profits. Buying more tickets (sending more messages) improves the odds of breakthrough, but reduces the chances for other senders. The formal model follows.

A single receiver receives $n$ anonymous messages, and will examine $\min \{\phi, n\}$ of them, so $\phi<n$ indicates information congestion. ${ }^{6}$ The assumption of a constant maximal $\phi$ is broadly consistent with some empirical evidence from the marketing literature. ${ }^{7}$ Our key equilibrium properties,

\footnotetext{
${ }^{5}$ The equilibrium is as follows. For $\frac{\pi}{\gamma} \leq 1$, neither advertiser sends; for $\frac{\pi}{\gamma} \in[1,2]$ one sends one message, and the other sends nothing; for $\frac{\pi}{\gamma} \in[2,6]$, each sends one message; and for $\frac{\pi}{\gamma} \geq 6$, both send two messages. There is also an equilibrium for $\frac{\pi}{\gamma}=6$ where one sends one message and the other sends two.

${ }^{6}$ Bounded rationality is incorporated here as a limit to the brain's capacity of processing information.

${ }^{7}$ Webb and Ray (1979) indicate a maximum number of TV ads that subjects can recall (around 4.2-4.5 per hour:
} 
such as the multiplicity of equilibria, survive under endogenous examination (it suffices to take an examination function with a zero marginal opening cost below $\phi$ and a prohibitive one above it).

We assume that if a message is examined, a further message from the same sender has no impact: two successful ads are no better than one. The benefit to a sender from multiple messages is a higher communication probability. We make this stark assumption to emphasize the motive for replication of ads even in a world where repetition has no intrinsic persuasion value.

There is a continuum of senders, with total mass $M$, and they are ranked by their expected profit from reaching a receiver with a message. A sender's rank (or type) is denoted by $\theta \in[0,1]$, with associated conditional profit $\pi(\theta)>0 .{ }^{8}$ We assume this profit is independent of which other messages are examined so that advertising does not change the nature of product market competition. This simplifying assumption allows us to concentrate on the congestion of messages in the receiver's attention span, without worrying about direct "business stealing" across messages. ${ }^{9}$ If there were competition within sectors, higher message costs might cause less competition between firms (for example, the model of Grossman-Shapiro, 1984, its elaboration by Cristou and Vettas, 2008, and an empirical setting in Sovinsky Goeree, 2008). In parallel research (Anderson and de Palma, 2012) we address this issue by considering sub-groupings of senders ("industries") that compete in the same product market as well as for attention in aggregate (although senders only transmit one message each in that model).

We assume $\pi(\theta)$ is continuously differentiable and strictly increasing with $\pi(0)<\gamma<\pi(1)$, where $\gamma>0$ is the cost of sending a message. These bounds on $\pi($.$) ensure the market is neither$ unserved nor completely covered. We can immediately deal with equilibrium with no congestion $(\phi \geq n)$. The proofs of all results are given in the Appendix.

Lemma 1 (i) Types $\theta<\pi^{-1}(\gamma) \equiv \theta_{\min }$ send no messages in any equilibrium. (ii) There is an

see their Table 2). Brown and Rothschild's (1993) experiments suggest there may be a less severe congestion effect at high levels of clutter. We emphasize the attention span bottleneck by assuming $\phi$ is constant, but we conjecture the main results still hold true if $\phi / n$ (which is the ad recall rate in the marketing studies) were decreasing in the number of messages, $n$, even if $\phi$ were increasing in $n$.

${ }^{8}$ The possibility that a consumer is not interested in the product is folded into the expected conditional profit.

${ }^{9}$ By contrast, in Butters (1977) all advertising messages received are examined, but only the lowest-priced one leads to profits. Here, only a limited number of messages are examined, but each message examined leads to positive expected profits for the sender. 
equilibrium with no congestion and all types $\theta \geq \theta_{\min }$ sending a single message if $\phi \geq\left(1-\theta_{\min }\right) M$.

Without congestion, a sender makes no profit if $\pi(\theta)=\gamma$, corresponding to the critical type, $\theta_{\min }$; hence (i). All senders with types above $\theta_{\min }$ send, so if the number of messages examined is larger than the number of such senders, there is no congestion, and no sender wants to send a second message; hence (ii).

We now deal with congestion. Suppose that a sender transmits an integer number $\ell \geq 1$ messages to the receiver. The probability that at least one of its messages is examined is given as follows. First, since messages are anonymous (their contents are unknown ex ante), the probability that a given message is examined is $\phi / n<1$. The probability that none of the $\ell$ messages is examined is $(1-\phi / n)^{\ell}$. Hence the breakthrough probability, $\mathbb{P}(\ell, \phi / n)$, that at least one of the sender's messages is examined is

$$
\mathbb{P}(\ell, \phi / n)=1-(1-\phi / n)^{\ell}
$$

which is an increasing and concave function of $\ell$.

Senders consider $n$ as fixed when choosing how many messages they should send. This is akin to a monopolistic competition assumption: each sender is a small contributor to the total volume of messages sent. Even though we show the individual sender's problem has a unique solution for a given total number of messages, differences in the anticipated number of messages change senders' choices and can thence support different equilibria. How many messages an advertiser should send depends on how much crowding there is from others.

The breakthrough probability (2) uses a specific micro-foundation, yet is consistent with other possible ways in which advertising repetition may work in generating sales. The probability (2) embodies a decreasing marginal effectiveness of a brand's ads, $\ell$, which concurs with the weight of the evidence from over 100 studies surveyed in Simon and Arndt (1980). ${ }^{10}$ Furthermore, (2)

\footnotetext{
${ }^{10}$ Other evidence suggests there may be an initial region of increasing returns to advertising (see for example Taylor, Kennedy, and Sharp, 2009.) Pechmann and Stewart (1988) suggest that wearin takes up to 3 ads, and wearout happens after around 15 ads. To capture such effects in our analysis, it suffices to renormalize messages in blocks of 3, so the first message in our set-up corresponds to 3 actual messages: an advertiser will not choose fewer than 3 if there are indeed initial increasing returns. In our model, different products have different optimal numbers of ads depending on the specific product and the overall congestion level.
} 
implies that effectiveness decreases with the overall amount of ad clutter, as suggested by evidence in many papers. For example, Hammer, Riebe, and Kennedy (2009) report recall rates for radio of $1.9 \%$ under low clutter (10 or fewer ads per half-hour) and $0.9 \%$ for high clutter (11 or more ads). Wind and Sharp (2009) give a set of empirical generalizations of the effects of ads: our approach melds a consumer model with an equilibrium model of firms.

\section{Existence of an equilibrium}

We construct the solution in 5 steps. Briefly, for a given $\theta_{1}>\theta_{\min }$, we first find the congestion level, $n / \phi$, needed to support $\theta_{1}$, then determine how many messages, $n$, are sent given the congestion level. We then back out the attention span required to support such a solution.

Step 1. Select a value of $\theta_{1} \in\left(\theta_{\min }, 1\right]$ and calculate congestion ratio

Here $\theta_{1}$ is the sender indifferent between sending one message or none. This marginal type is strictly worse off sending more than one message. Being indifferent, type $\theta_{1}$ makes zero profit, so

$$
\pi\left(\theta_{1}\right) \frac{\phi}{n}=\gamma
$$

while types $\theta<\theta_{1}$ do not send (Lemma 1 ). The (inverse) congestion ratio is $\frac{\phi}{n}<1$, so $\theta_{1}>\theta_{\min }$.

\section{Step 2. Calculate the number of messages sent by different senders}

Recalling that $\gamma$ is the cost of sending a message, sender $\theta$ 's problem is

$$
\max _{\ell=0,1,2, \ldots}\{\pi(\theta) \mathbb{P}(\ell, \phi / n)-\gamma \ell\}
$$

where we take explicit account of the constraint that the number of messages must be an integer. ${ }^{11}$

\footnotetext{
${ }^{11}$ The continuous problem can be derived from (4) in the linear profit case $(\pi(\theta)=\bar{\pi} \theta)$, and now treating $n$ as a continuous variable. The optimal number of messages for sender $\theta$ is $l(\theta)=-(1 / \omega) \ln [\gamma /(\omega \bar{\pi} \theta)]$, with $\omega=$ $-\ln (1-\phi / n)$. The lowest active sender type, $\underline{\theta}$, satisfies $l(\underline{\theta})=0$, so that $\underline{\theta}=\gamma / \omega \bar{\pi}$. Integrating $l(\theta)$ over $[\underline{\theta}, 1]$ to find $n(\underline{\theta})$ and then using $\pi \underline{\theta} \phi / n(\underline{\theta})=\gamma$ yields $\Phi(\underline{\theta})=(\underline{\theta} \bar{\pi}) / \gamma(\exp (-\gamma /(\underline{\theta} \bar{\pi}))-1)[1+\ln \underline{\theta}-\underline{\theta}]$ as the examination rate consistent with a marginal sender type of $\underline{\theta}$. It can be easily proved from this expression that there are two solutions (equilibria) for $\underline{\theta}$ for $\Phi$ low enough, but no solution for $\Phi$ large enough. This contrasts with the discrete model, which has an odd number of solutions for (almost all) $\Phi$. Although the continuous case is easier to solve than the discrete one, it is less satisfactory since the choice probabilities are not derived from an explicit micro-foundation. The lack of a solution for $\Phi$ too large is also problematic. This is the region (high examination) for which we should expect there to be no congestion in equilibrium. However, when $\phi=n$ (i.e., when congestion vanishes), the advertiser profit reduces to $\bar{\pi} \theta-\gamma l$, so that the optimal continuous number of messages is zero. This implies that the model will exhibit artifical discontinuities between the congestion to no congestion case. Imposing instead a minmum message
} 
Sender $\theta$ prefers to send $\ell$ rather than $\ell-1$ messages if the extra benefit of doing so exceeds the extra cost, i.e., if $\pi(\theta)[\mathbb{P}(\ell, \phi / n)-\mathbb{P}(\ell-1, \phi / n)]>\gamma$. That is, using (2):

$$
\pi(\theta)\left(1-\frac{\phi}{n}\right)^{\ell-1} \frac{\phi}{n}>\gamma, \quad \ell=1,2, \ldots
$$

The LHS is decreasing in $\ell$, indicating that the profit function is concave. With decreasing returns to messages sent, there are rents to higher $\theta$ senders. However, because $\pi(1)$ is finite, there is an upper bound to the maximum messages sent by an advertiser.

On the LHS of (5), $\left(1-\frac{\phi}{n}\right)^{\ell-1}$ is the probability that sender $\theta$ 's first $(\ell-1)$ messages are not examined, and $\frac{\phi}{n}$ (the inverse congestion ratio) is the probability that the last message (the marginal $\ell$-th one) is examined. Since the LHS increases in $\theta$, higher $\theta$ senders send out more ads.

The region $\left[\theta_{1}, 1\right]$ is partitioned into $k$ sub-intervals with advertisers in $\left(\theta_{j}, \theta_{j+1}\right)$ sending $j$ messages each. Advertiser $\theta_{j} \in(0,1)$ is indifferent between sending $j-1$ and $j$ messages, so

$$
\pi\left(\theta_{j}\right)=\frac{\gamma}{\left(1-\frac{\phi}{n}\right)^{j-1}} \frac{n}{\phi} .
$$

The RHS of this expression is increasing in $j$, so that $\theta_{1}<\theta_{2}<\ldots$ The number of messages sent is thus an increasing step-function of $\theta$ with unit step size. Substituting (3) into (6) yields an implicit expression for $\theta_{j}$ in terms solely of $\theta_{1}$ :

$$
\pi\left(\theta_{j}\right)=\frac{\pi\left(\theta_{1}\right)}{\left(1-\frac{\gamma}{\pi\left(\theta_{1}\right)}\right)^{j-1}} .
$$

As we will establish below, only one message will be sent in equilibrium if $\theta_{1}$ is either low or high enough; intermediate cases have more disparity across senders.

\section{Step 3. Find the maximal number of messages per sender}

The maximum number of messages per sender, denoted by $k$, is the number of messages sent by type $\theta=1$. Formally, $k$ is the largest integer such that $\theta_{k}$ defined in (7) lies below 1 . Hence $k$ is size of one also leads to discontinuities. We impute such problematic properties and differences to the unsatisfactory description afforded by the continuous approximation. 
the largest integer satisfying

$$
\frac{\pi\left(\theta_{1}\right)}{\left[1-\frac{\gamma}{\pi\left(\theta_{1}\right)}\right]^{k-1}} \leq \pi(1) .
$$

Taking logarithms, $k$ is the largest integer that satisfies $F\left(\theta_{1}\right)+1 \geq k$ with

$$
F\left(\theta_{1}\right) \equiv \frac{\ln \pi(1)-\ln \pi\left(\theta_{1}\right)}{\ln \pi\left(\theta_{1}\right)-\ln \left(\pi\left(\theta_{1}\right)-\gamma\right)}
$$

Equivalently, $k\left(\theta_{1}\right)=\left\lceil F\left(\theta_{1}\right)\right\rceil$ where $\lceil$.$\rceil denotes the ceiling function. Note that k-1$ is the amount of duplication of messages for the sender with the most messages (it sends $k$ ).

\section{Step 4. Aggregate message volume}

Given we have determined the number $(k)$ and position $\left(\theta_{j}\right)$ of steps, we can now determine the aggregate number of messages, $n$, for any given $\theta_{1}$. The number of messages transmitted is given by adding up the number of messages on each level, $j=1, \ldots k$, so

$$
n\left(\theta_{1}\right)=\left(1-\theta_{1}\right) M+\left(1-\theta_{2}\right) M+\ldots\left(1-\theta_{k}\right) M>0,
$$

with $n\left(\theta_{1}\right) \leq\left(1-\theta_{1}\right) M k$, so $n\left(\theta_{1}\right)$ is bounded. Therefore $n\left(\theta_{1}\right)=M k-M \sum_{j=1 \ldots k} \theta_{j}$, so that

$$
n\left(\theta_{1}\right)=M k-M \sum_{j=1 \ldots k} \pi^{-1}\left(\frac{\pi\left(\theta_{1}\right)}{\left[1-\frac{\gamma}{\pi\left(\theta_{1}\right)}\right]^{j-1}}\right) .
$$

\section{Step 5. Determine the level of examination consistent with $\theta_{1}$}

Define now $\Phi\left(\theta_{1}\right)$ as the value of $\phi$ that would support an equilibrium with $\theta_{1}$ as the marginal sender type. Using the value of $n\left(\theta_{1}\right)$ above, we can recover the value of $\Phi\left(\theta_{1}\right)$ from (3):

$$
\Phi\left(\theta_{1}\right)=\frac{n\left(\theta_{1}\right)}{\pi\left(\theta_{1}\right)} \gamma
$$

Note that $\Phi\left(\theta_{1}\right) \leq n\left(\theta_{1}\right)$ for $\theta_{1}>\theta_{\min }$, so that $\Phi\left(\theta_{1}\right)$ is bounded above in this case.

\section{Equilibrium existence}

Step 5 shows that there exists an attention span which supports an equilibrium characterized by any marginal type $\theta_{1}>\theta_{\text {min }}$. This attention span function, $\Phi\left(\theta_{1}\right)$ (given by (12)) allows us to 
back out the value or values of $\theta_{1}$ that can be sustained as equilibria for any $\phi$.

Lemma 2 For any $\theta_{1} \in\left(\theta_{\min }, 1\right]$, there exists a unique, continuous, and almost everywhere differentiable examination value $\Phi\left(\theta_{1}\right)$ given by (12) that supports a congested equilibrium with marginal sender $\theta_{1}$. As $\theta_{1} \downarrow \theta_{\min }$ or as $\theta_{1} \uparrow 1, k\left(\theta_{1}\right)=1$ and $\Phi\left(\theta_{1}\right)=\left(1-\theta_{1}\right) M$.

Equilibrium existence for $\phi \geq\left(1-\theta_{\min }\right) M$ follows from Lemma 1. For large enough $\phi$, this is the only equilibrium type because $\Phi$ is bounded on $\left(\theta_{\min }, 1\right]$. From Lemma $2, \Phi\left(\theta_{1}\right)$ is continuous on $\left(\theta_{\min }, 1\right]$ with limits $\left(1-\theta_{\min }\right) M$ and 0 . Hence any $\phi$ between these limits has a solution (or solutions) $\theta_{1} \in\left(\theta_{\min }, 1\right]$. By Lemma 2 , the limit solutions involve $k\left(\theta_{1}\right)=1$. Hence, we have:

Proposition 1 For any $\phi$ there exists an equilibrium. For $\phi$ large enough or small enough, there is a unique equilibrium, at which all active senders transmit only one message.

The function $\Phi\left(\theta_{1}\right)$ is not necessarily monotone, which gives rise to multiple equilibria.

\section{$5 \quad$ Illustrating multiple equilibria}

We use a linear profit function example, $\pi(\theta)=\bar{\pi} \theta$, to illustrate. Then (11) reduces to:

$$
n\left(\theta_{1}\right)=M k-M \theta_{1} \sum_{j=1 \ldots k}\left[1-\frac{\gamma}{\bar{\pi} \theta_{1}}\right]^{1-j} .
$$

Using then (12) determines the function, $\Phi\left(\theta_{1}\right)$, which is plotted in Figure 1 using the parameter values $\bar{\pi} / \gamma=20$ and $M=1$. (The curve continues up the vertical axis at $\theta_{1}=\theta_{\min }=0.05$ ).

To find the equilibrium from the attention span, suppose we start from an attention span just over 0.8. Then we simply read off the Figure that the value of $\theta_{1}$ is 0.2 , and that is the only equilibrium (the examination function only takes the value of 0.8 once). However, if the examination rate is 1.29 (the horizontal line in the Figure), then there are 5 possible equilibria.

For any equilibrium, we can determine the steps of the different message levels. This is done in Figure 2, which displays the critical $\theta$ 's as a function of $\theta_{1}$ using (7) where $\bar{\pi} / \gamma=20$ as in Figure 1. Think of the loci in Figure 2 as a contour map of the steps, with the steps increasing one step at a time in a north-north-easterly direction. Level zero is the lower-right triangle. 


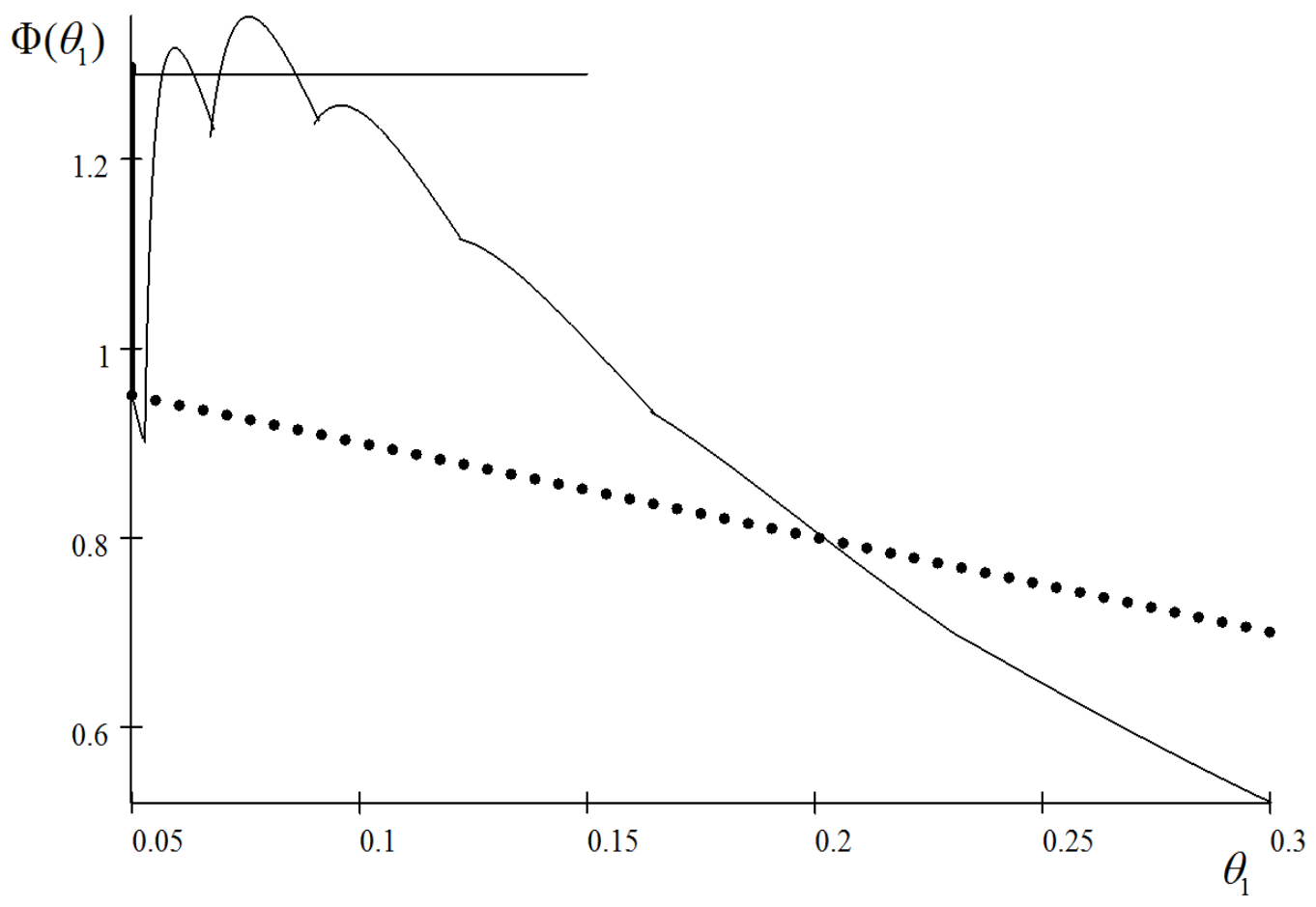

Figure 1: The function $\Phi$ (and the locus $\phi=1-\theta_{1}$ ).

Choose an equilibrium value of $\theta_{1}$ read off Figure 1. Figure 2 gives then the number of senders at each step level of messages. The black line (45 degree line) in Figure 2 is $\theta_{1}$, and denotes the lowest sender type of one message. The next line up (red) is the step-level locus for $j=2$ messages, followed by $j=3$ (dark green), etc. For example, consider a candidate solution with $\theta_{1}=0.4$. Then, high-profit senders with $\theta$ between 0.8 and 0.9 send 6 messages, and the maximum number of messages reaches 7 for all sender types above (approximately) 0.9. For the example given earlier, with an examination rate of just over 0.8 , we can read off the message step-levels in Figure 2 by proceeding vertically up the picture at $\theta_{1}=0.2$. The highest step level is six (the grey line), while seven (the blue line) is not attained.

The pattern in Figure 2 illustrates the property indicated in Proposition 1 that only one message will be sent in equilibrium if $\theta_{1}$ is either low or high. In conjunction with Figure 1, only one message is sent for high $\phi$ (little congestion and many senders) and also for low $\phi$ (high congestion and few 


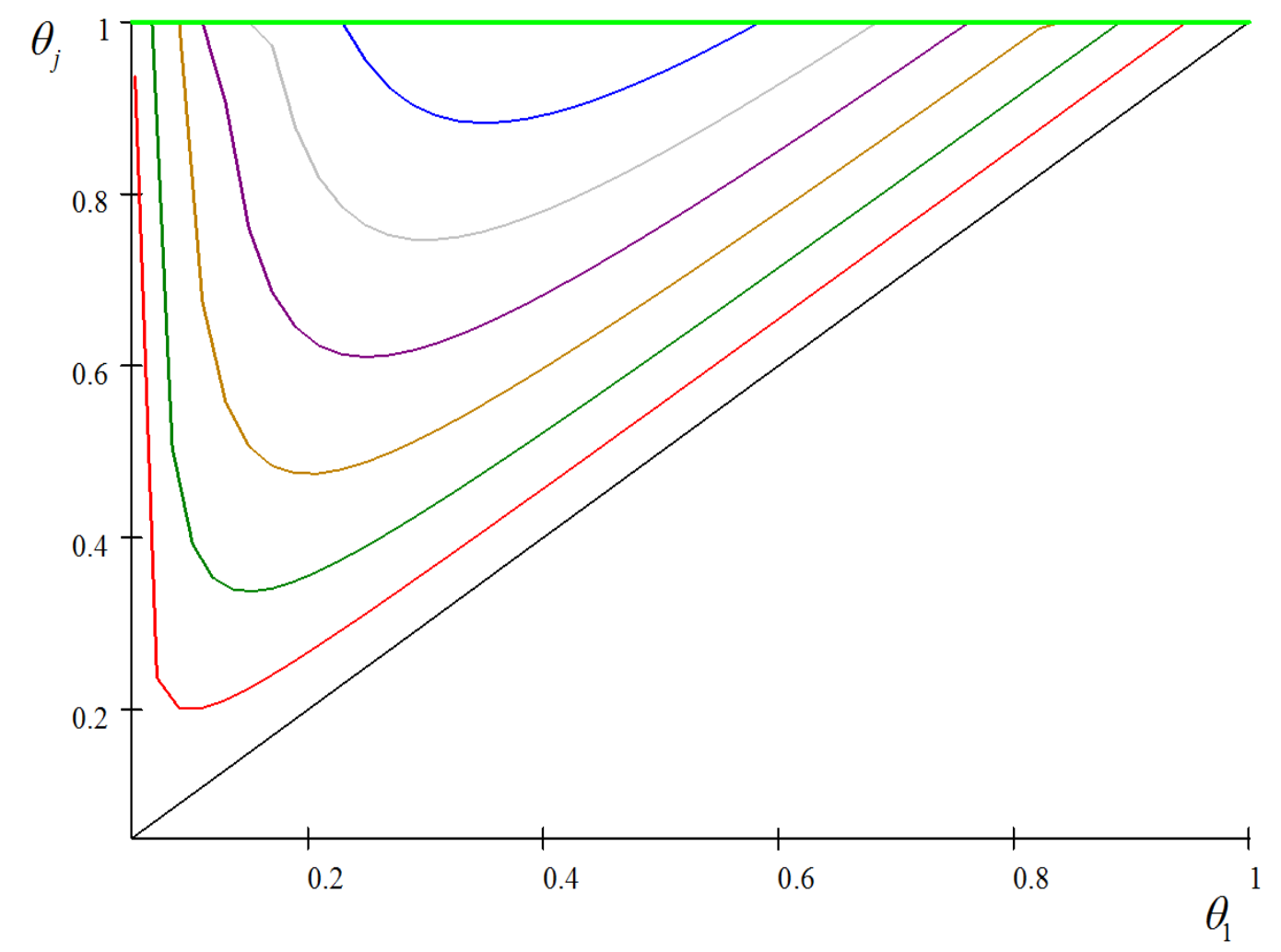

Figure 2: Critical step values, $\theta_{j}, j=1, \ldots, 7$. Red is lowest $\theta$ for 2 steps, green for 3 steps, etc.

senders). Intermediate cases have more disparity across senders. In the middle there are many different per sender message levels, corresponding to a large degree of endogenous differentiation across sender types. The higher sender types shout more to break through the others' clutter.

Note that the $\theta_{j}$ 's are single-troughed functions of $\theta_{1}$, which is a general property of these functions. It is shown in the Appendix (Property 1) that the maximum number of steps (i.e., different numbers of messages sent across senders) is for middling values of $\theta_{1}$. Thus the greatest disparities in message levels occurs for a middling width of senders. The maximal number of steps is seen from Figure 2 to be first increasing and then decreasing in $\theta_{1}$ (from 1 to 6 and then back down to 1: track the light green line at the top of the Figure). With $\theta_{1}$ small the marginal sender has a low profitability, and can only be induced to send a message if there is very little congestion, which in turn means that other senders do not want to send many messages because there is a high 
chance the first one will get through. At the other extreme, if the marginal sender is high, then there are few higher types - they are unlikely to want to send several messages given the marginal type did not (unless the profit as a function of type becomes very elastic). ${ }^{12}$

\section{Properties of equilibrium}

Figure 1 shows an odd number of equilibria for almost all $\phi$. More generally, Lemmas 1 and 2 show that $\Phi$ is continuous on $\left(\theta_{\min }, 1\right]$. At $\theta_{1}=\theta_{\min }, \Phi$ takes all values greater than or equal to $\left(1-\theta_{\min }\right) M$, and at $\theta_{1}=1, \Phi=0$. Hence, any value of $\phi$ cuts the function $\Phi\left(\theta_{1}\right)$ an odd number of times, except when $\phi$ corresponds to a turning point of $\Phi$.

\subsection{More volume with fewer shouting}

The main characterization result of different equilibria is that there is more shouting to be heard (more congestion) at equilibria with fewer senders:

Proposition 2 Consider a set of equilibria, for given $\phi$, ranked from low to high values of the marginal sender type, $\theta_{1}$. Then the maximal number of messages per sender, $k$, is weakly increasing in $\theta_{1}$ and more messages are sent. Profits of each active sender and aggregate profits are strictly decreasing in the equilibrium value of $\theta_{1}$.

The intuition for multiplicity of equilibria and the properties in the Proposition is already seen from the simple matrix game in Section 2 where there can be two equilibria because of strategic complementarity in message level choices. There, the best reply to one message was one, but the best reply to two was two, so both are equilibria. The full-fledged model is more intricate. Indeed, it is readily shown from analyzing the marginal condition for choice of message level, (5), that messages are strategic substitutes for low-volume senders and strategic complements for highvolume ones. ${ }^{13}$ This means that as the message level rises, small senders send less, or are forced

\footnotetext{
${ }^{12}$ With high $\theta_{1}$, there must be a relatively high congestion level, which at first glance would seem to suggest a high level of messages per sender. However, recall that a high level of $\theta_{1}$ is induced by a low attention span. The high congestion level results from a low attention span despite a low volume of messages sent.

${ }^{13}$ The derivative (with respect to $n$ ) of the incremental benefit in $(5)$ has the sign of $1-(\ell+1) \frac{\phi}{n}$, and so this is negative for large $\ell$ and positive for small $\ell$.
} 
out by the noise from above, while large senders respond by sending more, and indeed, so much more that another equilibrium can sustain with greater volume.

From the Proposition, either there is the same number of levels of message-sending (same $k$ ) as we consider equilibria with higher $\theta_{1}$, and the $k^{\text {th }}$ level kicks in earlier (i.e., at a lower level of $\theta$ ); or else the number of messages on the top step is bigger than before. Both cases lead to a higher number of messages sent.

For a large enough sending cost, Proposition 1 shows there is but one equilibrium. Applying Property 2 in the Appendix, the equilibrium involves one message per sender (with some active senders) if $\gamma \in\left(\frac{\bar{\pi}}{4}, \bar{\pi}\right)$ and profits are linear. Some further properties of the model are given in the Appendix for a linear profit function.

\subsection{Step-size relations}

We now show some properties of the width of ad step levels (how many senders choose each intensity of transmission). From (7), taking logs, we can write

$$
\ln \pi\left(\theta_{j}\right)=\ln \pi\left(\theta_{1}\right)+(j-1) \ln \left(1-\frac{\gamma}{\pi\left(\theta_{1}\right)}\right)^{-1},
$$

so that $\ln \pi\left(\theta_{j}\right)$ is seen to be a linear increasing function of $j$. We will argue that the step-size relation depends on the concavity or convexity of $\ln \pi(\theta)$. To see this simply, first suppose that $\ln \pi(\theta)$ is a linear function (equivalently, $\pi(\theta)$ is an exponential function). Then each step width has the same size because each increment in $j$ has the same increment in $\ln \pi(\theta)$ and hence it has the same increment in $\theta_{j}$ ( $\operatorname{since} \ln \pi(\theta)$ is linear). Now suppose that $\ln \pi(\theta)$ is convex (equivalently, $\pi(\theta)$ is log-convex, meaning "more convex" than an exponential function). Then each increment in $j$ has the same increment in $\ln \pi(\theta)$ but then, by convexity, the corresponding increment in $\theta_{j}$ gets smaller and smaller as $j$ rises. This means that the steps become narrower: increasingly fewer senders are at higher message levels. Concavity of $\ln \pi(\theta)(\pi(\theta) \log$-concave) has the opposite impact. To summarize:

Proposition 3 Step widths decrease with step level if $\ln \pi(\theta)$ is convex, and increase if $\ln \pi(\theta)$ is concave. 
The uniform distribution used in the earlier examples is a log-concave distribution. Accordingly, as can be seen from Figure 2, step sizes get wider going up the stairs, and similarly for any logconcave function. Conversely, for log-convex $\pi(\theta)$ functions, there are more senders of one message than there are of two messages, than there are of three, etc. This is reminiscent of the Long Tail popularized for the internet by Anderson (2006). It would be interesting to derive the size distribution of messages sent from the underlying economic primitive, which here is the distribution of profitability. Already, the idea of the long-tail is present in the idea that there may be many small senders and few shouters. However, one key feature missing from the model (and thus for future work) is the agility and ability the internet affords for targeting of messages; this feature may accentuate Long-Tail properties.

\subsection{Narrow sender range means high congestion}

Figure 1 shows that changes in the attention span can change the equilibria in different directions in a local sense. Nonetheless, there are still some strong properties that follow from comparing equilibria. In particular, we now show congestion must rise around the attention span function, despite its bumpiness. Hence a narrower width of senders is associated to more congestion.

Recall (from (12)) that $n\left(\theta_{1}\right) / \Phi\left(\theta_{1}\right)=\pi\left(\theta_{1}\right) / \gamma$. Hence the congestion rate $n\left(\theta_{1}\right) / \Phi\left(\theta_{1}\right)$ is strictly increasing in $\theta_{1}$ along the curve $\Phi\left(\theta_{1}\right)$ since $\pi\left(\theta_{1}\right)$ is strictly increasing in $\theta_{1}$. Even though the function $\Phi\left(\theta_{1}\right)$ may be locally increasing, the corresponding increase in $n$ is always large enough to raise the congestion rate, $n\left(\theta_{1}\right) / \Phi\left(\theta_{1}\right)$, as $\theta_{1}$ rises. Thus if a more profitable sender (one with a higher $\theta$ ) is the marginal one, congestion will be higher even though there are fewer senders!

The surprising result arises if the attention span function, $\Phi($.$) , is locally increasing. Then an$ increase in $\phi$ locally raises the total number of messages sent and the congestion rate, meaning that the extra volume of messages submerges the greater attention. However, if $\Phi($.$) is locally$ decreasing, a larger attention span eases congestion and allows in lower sender types. 


\section{$7 \quad$ Advertising costs and ad caps}

\subsection{Raising the cost of sending messages}

Surprisingly, there may be significant benefits to ALL active senders from higher message transmission costs because of the reduction in "shouting." 14 We now show when this is possible.

We first recall from Lemma 1 (ii) that a marginal sender type $\theta_{1}=\theta_{\min }$ can be supported as an equilibrium if and only if $\phi \geq\left(1-\theta_{1}\right) M$. Such an equilibrium entails a transmission cost $\gamma=\pi\left(\theta_{1}\right)$ and $k=1$, i.e., one message per sender. In Figure 1, the dotted line represents the equation $\phi=\left(1-\theta_{1}\right) M \cdot{ }^{15}$ Any $\left(\phi, \theta_{1}\right)$ below it must have congestion, even if senders sent only one message. Above or on it, there would be no congestion if senders only sent one message. In Figure $1, \Phi$ is vertical at $\theta_{\min }$ down to its intersection with the line $\phi=\left(1-\theta_{1}\right) M$. The experiment of the next sub-section takes an equilibrium point above this line, and effectively drops it down to the line by raising $\gamma$. A simple condition ensures all senders can be better off than at an equilibrium.

Proposition 4 Consider a congested equilibrium with marginal sender type $\theta_{1}$. It is possible without lump-sum transfers to make almost all active senders better off by raising the transmission cost to $\hat{\gamma}=\pi\left(\theta_{1}\right)\left(=\frac{n}{\phi} \gamma\right)$ if $\left(1-\theta_{1}\right) M \leq \phi$.

If instead $\phi<\left(1-\theta_{1}\right) M$, it is not possible to have all active senders transmit a message and have them all read. It may though still be possible to make all senders better off. ${ }^{16}$ For the case of the Proposition, the receiver would still examine more messages than the number actually sent (if $\phi>\left(1-\theta_{1}\right) M$ ), and so further welfare gains could be realized. It is striking that any higher profits from higher transmission costs need not be redistributed for everyone to be better off.

Van Zandt (2004) also finds that all senders can be better off, although the mechanism is quite different. In his paper, messages are targeted to receivers with diverse preferences (and each sender transmits a single message). Gains from raising sending costs arise because marginal senders are eliminated from receivers where they are less profitable, so benefiting those remaining through

\footnotetext{
${ }^{14}$ This is also true in Grossman and Shapiro (1984) where higher costs induce less market overlap and so higher oligopoly prices. Here the mechanism is through reduced congestion rather than through relaxed price competition.

${ }^{15}$ This locus represents the social optimum for senders: no congestion and the $\phi$ highest profit types sending.

${ }^{16}$ To see this, it suffices to note that when there are multiple equilibria we could move to an equilibrium with less congestion even while leaving $\gamma$ unchanged.
} 
reduced congestion, and eliminated senders benefit on other receivers where they have a profit advantage. Here it is multiple messages that are pruned, even though there is a single receiver type.

One important extension for future research is to take $\gamma$ as endogenous: for example, the outcome of bidding by prospective advertisers for slots on web-pages (see e.g., de Corniere, 2011). The simplest case to analyze is when $\gamma$ is set by a monopolist (say the Post Office for bulk mail). Tentative results suggest that a monopolist will price out congestion (Anderson and de Palma, 2009, prove this when firms send but one message) and so the solution is simply the monopoly price against the demand curve generated by $\pi(\theta)$.

\subsection{Ad caps (and conditional examination functions)}

To describe ad caps, we construct $\Phi\left(\theta_{1}\right)$ from its component pieces. We show that $\Phi\left(\theta_{1}\right)$ is the upper envelope of sub-functions $\Phi_{i}\left(\theta_{1}\right)$ defined by modifying (12) to

$$
\Phi_{i}\left(\theta_{1}\right) \equiv \frac{n_{i}\left(\theta_{1}\right)}{\pi\left(\theta_{1}\right)} \gamma
$$

where $n_{i}\left(\theta_{1}\right)$ is the number of messages sent under the restriction that each sender can send no more than $i$ messages (cf. (10):

$$
n_{i}\left(\theta_{1}\right)=\left(1-\theta_{1}\right) M+\left(1-\theta_{2}\right) M+\ldots\left(1-\theta_{i}\right) M, \quad i \leq k\left(\theta_{1}\right) .
$$

In terms of the 5-step procedure, the functions $\Phi_{i}\left(\theta_{1}\right)$ are given by skipping step 3 (which endogenously determined $k$ ). The functions $\Phi_{i}\left(\theta_{1}\right)$ can therefore be viewed as those of a constrained problem, where at most $i \leq k$ messages per sender are allowed.

For a given $\theta_{1}$, a higher value of the maximal number of steps allowed, $i$, implies that the corresponding total volume of messages sent, $n_{i}\left(\theta_{1}\right)$, is (weakly) higher (because the lower- $\theta$ senders are unchanged but the high- $\theta$ ones send more - see (15)). Given that the congestion level is fixed by the initial choice of $\theta_{1}$, more messages support more examination (recall (14)). Since $k$ is the highest possible value of $i$ for any $\theta_{1}$, the level of examination that will support an equilibrium with marginal sender type $\theta_{1}$ is at the highest level, $k$, i.e., $\Phi\left(\theta_{1}\right)=\Phi_{k}\left(\theta_{1}\right)$. In summary, 
Lemma $3 \Phi\left(\theta_{1}\right)$ is the upper envelope of the conditional examination functions $\Phi_{i}\left(\theta_{1}\right), i=1, \ldots, k$.

The intuition why the highest conditional examination function is the relevant one is as follows. Suppose we were to select a lower level for the maximum number of messages examined. Then, given the purported $\left(\theta_{1}, \phi\right)$ pair, the top firm $(\theta=1)$ would want to send more messages. Letting it do so raises the congestion level, which can only be reduced to its previous level (in order to keep the lowest type, $\theta_{1}$, still active) by reducing congestion again, that is, raising the attention span. Figure 3 illustrates (with the same parameter values as before). The colored relations are the conditional examination functions corresponding to different levels of examination by the highestprofit sender $(\theta=1)$, and the full examination function is the upper envelope of these (this gives Figure 1). The black line is the conditional examination function for a one-message maximum. This would be the only curve were we to constrain senders to send out just one message. Red denotes a two-message maximum, green is three, sienna four, purple five, grey six, and blue seven (the colors are coordinated with Figure 2).

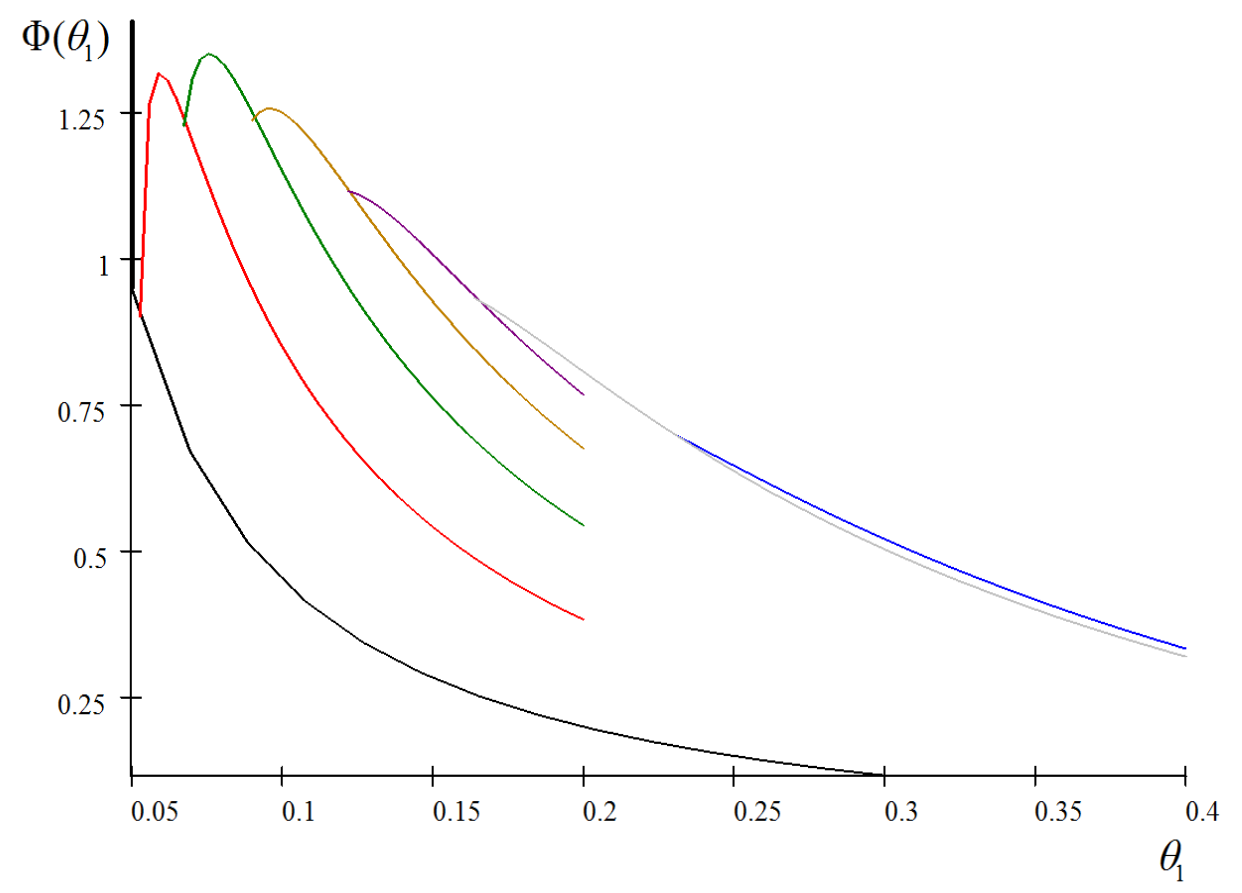

Figure 3: The functions $\Phi_{i}$. 
The impact of message caps can be seen from Figure 3. For example, if $\phi$ is slightly bigger than 1.25, the number of equilibria drops from 5 to 3 with a cap of 2 messages per sender. As per Proposition 2, sender welfare rises if the equilibrium in the absence of a cap had some senders transmitting 3 messages. However, if $\phi=0.6$, say, then the only equilibrium had up to 7 messages transmitted per sender. If a cap of 2 messages per sender were imposed, then the new equilibrium would be at the value of $\theta_{1}$ where the red curve has a value of 0.6 on its descending part. More senders would transmit, because of less crowding by the louder shouters, which gives a clear gain to low- $\theta$ types - some of these become more profitable and enter the market, while others benefit directly from the reduction in congestion. However, the erstwhile loud shouters (high $\theta$-types) have less chance of breaking through the clutter and therefore may be worse off. Despite less congestion, they now are constrained by the cap to send only two messages and thus have a lower chance of getting their very profitable message across. Thus the gains from less congestion and a broader sender base may be offset by the loss of breakthrough probability to the most profitable types.

\section{Conclusions}

When consumer attention spans are congested, advertisers with stronger benefits from getting across their messages will send more messages. This feature implies that advertisers with higher benefits do at least get a better chance of getting attention. Achieving prominence from multiple messages is socially desirable at least insofar as the social ranking of messages follows the private ranking. The private ranking is by profitability, but this may not necessarily reflect the social benefits, as we explain below. Holding aside that issue for the moment, we note that a regulated cap on the number of messages that can be sent may have the adverse effect of closing down the ability of higher-profit advertisers to be more prominent. This aspect is to be traded off against the benefit from a cap of reducing congestion.

We have modeled congestion as a fixed pipe of messages examined from the total number sent. A useful extension would be to provided a finer micro-model of consumer choice. One promising direction is to consider optimal consumer choice along the lines of Kuksov and Villas-Boas (2012). These authors consider a sequential-choice search model with the intriguing finding that a wider 
choice set can actually make consumers worse off via reducing the expected benefits from search. Then, a congestion effect arises because mismatches increase. This has a parallel in the model of Anderson and de Palma (2009) in which the optimal number examined can fall with more sent because the average quality of messages falls if consumer surplus and message profitability are positively correlated.

We now briefly address receiver welfare. The critical point to note is that the market equilibrium is driven by sender profitability from sending messages, whereas the optimum message pattern also accounts for receiver benefits. In an extreme case, we can imagine that firm profits are diametrically opposed to receiver benefits (Nigerian spam emails spring to mind). Because the high profit advertisers shout a lot to get a high likelihood from being seen, they may completely crowd out messages that the receiver would have been interested in. Consumers are then very likely to get messages about products they do not want. If the sum of surplus to receivers plus benefits to senders also follows the opposite ranking from the ranking of profits, then the profit criterion again may crowd out the socially desirable products, or at least give most prominence to less desirable ones. Here shouting exacerbates the problem in the market mechanism.

Matters are more subtle if receivers and senders have the same ranking of products, which happens if the surplus to receivers is rising with $\theta$ (along with the profits to senders), as in Anderson and de Palma (2009). Even then, a comparison of receiver welfare across equilibria is not straightforward. If consumer benefits are strongly increasing in $\theta$, then an equilibrium with small width but a large number of messages sent by high $\theta$ types (and hence a high probability that the most desired messages get through) might be preferred by receivers to one with large width and little congestion (because still the most desired messages might be crowded out).

To shed further light on the bias in the market mechanism, suppose that consumer surplus rises with profit, but at a slow rate (double the profit means less than double the receiver surplus, say). Then, there will be a market bias towards high profit senders who shout too much. Even though a lot of shouting is good insofar as it gets over the message better when there are high surpluses, it overdoes it if the total surplus (consumers plus firms) rises slower than the profits. Conversely, if surplus rises faster than profits there is not enough shouting at the top in the sense that there is 
too small a chance the most socially desirable messages get through.

Johnson (2011) also considers situations where social and private incentives to advertise may be misaligned. In his second model of targeted advertising, firms bid for the right to show an ad to heterogeneous consumers. Depending on the surpluses to various parties, the "wrong" firms may show the ad (this is analogous to excess shouting). Moreover, improved targeting may or may not improve consumer welfare, so that welfare results are quite subtle in his model too.

Everything in the current model is static, so that yesterday's decisions do not affect today's purchases. This may be viewed as daily receipt of junk mail, with consumers each day forgetting yesterday's offers. It may also be relevant to TV-watching with transient commercials that are already out of one's consciousness by the next break. Dynamic models of memory retention, advertising pulsing, and churn in ads as consumers take up offers would be clearly desirable.

Brands may be an important sorting device for receivers to pay attention to messages. It would be useful to allow consumers to pay more attention prominence to messages of recognized brands.

The framework here can be adapted to allow for targeting of advertising, for example by tracking internet sites visited (for more on this topic, see Bergemann and Bonatti, 2011, Johnson, 2011, and Iyer, Soberman, and Villas-Boas, 2005). We treat a single receiver, but the results apply to different receivers too under targeting. Some results are quite immediate under targeting individuals of different profiles. For example, suppose individuals vary only by their examination values, $\phi$. Suppose too that the equilibrium involves the maximal number of messages, and so is on the downward-sloping part of the function $\Phi\left(\theta_{1}\right)$ (see Figure 1). A higher $\phi$ then entails a lower $\theta_{1}$, so the congestion rate $(n / \phi)$ is lower for individuals examining more. Results for more elaborate patterns of individual differences remain a subject for further work. 


\section{Appendix (proofs)}

Proof of Lemma 1: (i) Profit of type $\theta$ is at most $\pi(\theta)-\gamma$ because its profit is greatest if its message was examined for sure. This profit is negative for $\theta<\theta_{\min }$.

(ii) If $\phi \geq n$ then all messages are examined. There are $n=\left(1-\theta_{\min }\right) M$ senders with type $\theta$ above $\theta_{\min }$ and so if all types $\theta$ with $\pi(\theta) \geq \gamma$ send a single message, all their messages are examined (so there is no point to sending further messages), and they all make non-negative profits if the condition holds. Q.E.D.

Property 1: Consider a candidate equilibrium with all senders $\theta \geq \theta_{1}$ active. The maximum duplication $k-1$ first increases and then decreases with $\theta_{1}$. Duplication tends to zero when $\theta_{1} \downarrow \theta_{\min }$ or $\theta_{1} \uparrow 1$.

Proof: We show below that $F\left(\theta_{1}\right)$ is quasi-concave and tends to zero at both limits $\theta_{1} \downarrow \theta_{\text {min }}$ and $\theta_{1} \uparrow 1$, and so $k\left(\theta_{1}\right)$ is unity approaching these limits. Hence, either the number of steps, $k$ is always unity, or else it increases and then decreases with $\theta_{1}$. Indeed, the maximum number of messages, $k$, sent by any sender for any $\theta_{1}$ is determined in the text as $k\left(\theta_{1}\right)=\left\lceil F\left(\theta_{1}\right)\right\rceil$ with $F\left(\theta_{1}\right)=\frac{\ln \pi\left(\theta_{1}\right)-\ln \pi(1)}{\ln \left(1-\frac{\gamma}{\pi\left(\theta_{1}\right)}\right)}($ see $(9)$ and Figure 2).

We now prove that this function is quasi-concave in $\theta_{1}$. At the end-points we have $F\left(\theta_{\min }\right)=0$ and $F(1)=0 . \quad F($.$) is also continuously differentiable and positive for \theta_{1} \in\left(\theta_{\min }, 1\right)$; therefore there is a least one turning point. We show that there is a unique solution to $F^{\prime}\left(\theta_{1}\right)=0$ so that $F\left(\theta_{1}\right)$ is quasi-concave on its support. Differentiation of $F($.$) implies that$

$$
\operatorname{sgn}\left[F^{\prime}\left(\theta_{1}\right)\right]=\operatorname{sgn}\left[\frac{\pi\left(\theta_{1}\right)}{\gamma}\left(1-\frac{\gamma}{\pi\left(\theta_{1}\right)}\right) \ln \left(1-\frac{\gamma}{\pi\left(\theta_{1}\right)}\right)-\ln \pi\left(\theta_{1}\right)+\ln \pi(1)\right] .
$$

Define $x=\gamma / \pi\left(\theta_{1}\right)$, with $x \in(0,1)$. Then we can rewrite

$$
\operatorname{sgn}\left[F^{\prime}\left(\theta_{1}\right)\right]=\operatorname{sgn}\left[\left(\frac{1}{x}-1\right) \ln (1-x)-\ln \pi\left(\theta_{1}\right)+\ln \pi(1)\right] .
$$

Any solution to $F^{\prime}\left(\theta_{1}\right)=0$ solves the equation

$$
\left(\frac{1}{x}-1\right) \ln (1-x)=\ln \pi\left(\theta_{1}\right)-\ln \pi(1)
$$




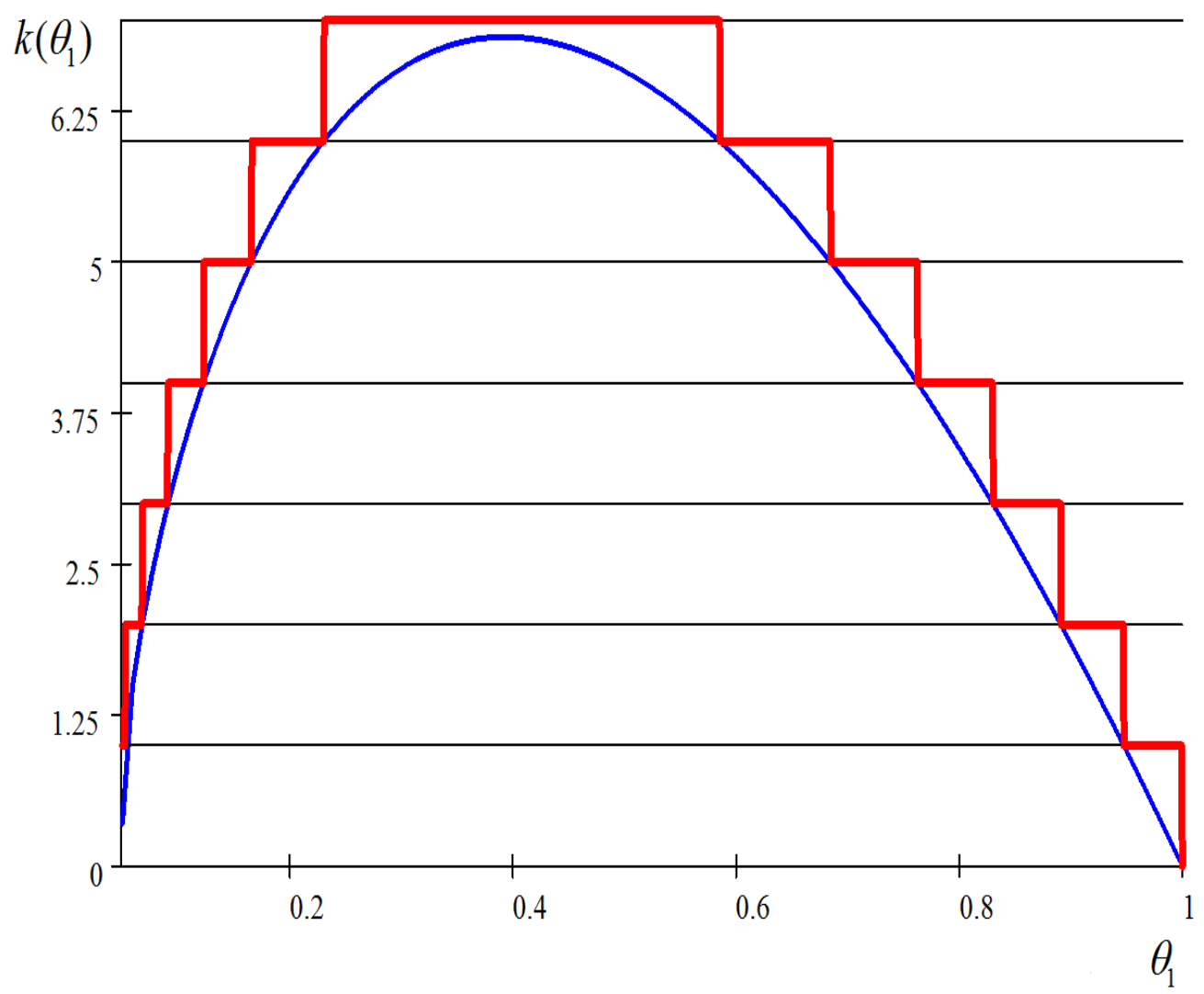

Figure 4: The function $F\left(\theta_{1}\right)$ (blue) and its ceiling function $k\left(\theta_{1}\right)$ (red) for $\bar{\pi} / \gamma=20$.

The RHS of this equation is increasing in $\theta_{1}$. For the solution to $F^{\prime}\left(\theta_{1}\right)=0$ to be unique, it then suffices to prove that the LHS in increasing in $x$ (since $x$ is decreasing in $\theta_{1}$ ). Here

$$
\frac{d}{d x}\left[\left(\frac{1}{x}-1\right) \ln (1-x)\right]=-\ln (1-x)-x>0,
$$

since $-\ln (1-x)-x$ is increasing and zero at $x=0$. Quasi-concavity and continuity of $F($. implies that the maximal number of messages sent is either always 1 or else is increasing and then decreasing in steps of size 1. Q.E.D.

The function $F\left(\theta_{1}\right)$ and the construction of the associated ceiling function, $k\left(\theta_{1}\right)$, is given in Figure 4 for the uniform profit example, $\pi(\theta)=\bar{\pi} \theta$.

Proof of Lemma 2. Existence of a unique solution is given by the 5 -step procedure in the text (see 
expressions (8), (11), and (12)). The key is that any $\theta_{1}>\theta_{\min }$ induces a value of $\Phi\left(\theta_{1}\right)=\frac{n\left(\theta_{1}\right)}{\pi\left(\theta_{1}\right)} \gamma$ which is bounded above.

Because $\pi\left(\theta_{1}\right)$ is positive and continuous, to show $\Phi\left(\theta_{1}\right)$ is continuous is proved by showing that $n\left(\theta_{1}\right)$ is continuous. Define a regime by its corresponding value of $k$. The boundary between two regimes as the uppermost number of messages transmitted changes between $k$ and $k-1$ is given by $\theta_{k}=1$. Continuity and differentiability within each regime (i.e., for given $k$ ) follows from the fact that each of the 5 steps preserves continuity and differentiability. Now consider the boundaries between regimes. In regime $k$ with $\theta_{k}=1$, we have (from (10)): $n\left(\theta_{1}\right)=\left(1-\theta_{1}\right) M+\left(1-\theta_{2}\right) M+$ $\ldots\left(1-\theta_{k-1}\right) M$, which is clearly equal to the expression for $n\left(\theta_{1}\right)$ in the regime $k-1$. Since the functions (7) defining $\theta_{k}$ as functions of $\theta_{1}$ are continuous in $\theta_{1}$, it is necessarily the case that continuity of $n\left(\theta_{1}\right)$ is preserved between neighboring regimes. Therefore $n\left(\theta_{1}\right)$ is also continuous across regimes. However, it is not generally differentiable at the critical points corresponding to the boundary between $k$ and $k-1$.

The last part is shown as follows. First we prove that $\lim _{\theta_{1} \downarrow \theta_{\min }} \Phi\left(\theta_{1}\right)=\left(1-\theta_{\min }\right) M$. Let $\pi\left(\theta_{1}\right)=\gamma+\varepsilon, \varepsilon>0$. Then we have the following inequalities from the bounds defining $k$ (see (8)):

$$
\frac{\gamma+\varepsilon}{\left(1-\frac{\gamma}{\gamma+\varepsilon}\right)^{k-1}} \leq \pi(1)<\frac{\gamma+\varepsilon}{\left(1-\frac{\gamma}{\gamma+\varepsilon}\right)^{k}},
$$

for which the only solution for $\varepsilon$ small enough is $k=1$ (recall that $\pi($.$) is continuous and that$ $\gamma<\pi(1))$. Given $k=1$, then the number of messages sent tends to $n\left(\theta_{\min }\right)=\left(1-\theta_{\min }\right) M$. Furthermore, by (3), the congestion rate, $n / \phi$, is unity (meaning all messages sent are examined). Thus $\phi$ must also tend to $\left(1-\theta_{\min }\right) M$, as was to be proven.

We next show that $\lim _{\theta_{1} \uparrow 1} \Phi\left(\theta_{1}\right)=0$. In this case, we have $\pi\left(\theta_{1}\right)=\pi(1)-\varepsilon$, for $\varepsilon>0$. The inequalities defining $k$ are now:

$$
\frac{\pi(1)-\varepsilon}{\left(1-\frac{\gamma}{\pi(1)-\varepsilon}\right)^{k-1}} \leq \pi(1)<\frac{\pi(1)-\varepsilon}{\left(1-\frac{\gamma}{\pi(1)-\varepsilon}\right)^{k}}
$$

which clearly has a unique solution $k=1$ for $\varepsilon$ small enough. Therefore $n\left(\theta_{1}\right)=\left(1-\theta_{1}\right) M$ and $n\left(\theta_{1}\right) \downarrow 0$ as $\theta_{1} \uparrow 1$. Since $\phi<n, \phi$ must also tend to 0 as $\theta_{1} \uparrow 1$, by (3). Q.E.D. 
Proof of Proposition 2. From (3) we have $n=\phi \pi\left(\theta_{1}\right) / \gamma$, so that higher $\theta_{1}$ imply higher $n$ since $\phi$ is given. Thus, there is a higher amount of congestion (more "shouting to be heard"). We now show that the corresponding $k$ cannot decrease. Differentiating (7) shows that $\frac{d \theta_{j}}{d \theta_{1}}$ has the same sign as $\left[1-\frac{j \gamma}{\pi\left(\theta_{1}\right)}\right]$. Hence, if $\frac{d \theta_{j}}{d \theta_{1}}>0$, then $\frac{d \theta_{i}}{d \theta_{1}}>0$ for all $i<j$. However, if $\frac{d \theta_{k}}{d \theta_{1}}>0$, all the values of $\theta_{j}$ have increased and there must be fewer messages sent for the higher level of $\theta_{1}$, a contradiction. Hence it must be that $\frac{d \theta_{k}}{d \theta_{1}}<0$, and at least as many message levels must be present to generate a larger message volume at higher $\theta_{1}$. The last result follows from the property that equilibrium profits per sender decrease in $n$ (see (4) and applying the envelope theorem); the aggregate result then follows directly. Q.E.D.

Property 2. If $\gamma \geq \frac{\pi(1)}{4}$, then for any $\phi$ there is only one equilibrium: in equilibrium, only one message is sent by each active sender. If $\gamma<\frac{\pi(1)}{4}$ there exists some $\phi$ for which an equilibrium exists with more than one message being sent by some senders.

Proof: Recall first from (8) that if $\pi(1) \leq \frac{\pi\left(\theta_{1}\right)}{1-\frac{\gamma}{\pi\left(\theta_{1}\right)}}$ then at most one message will be sent (the sender $\theta=1$ is just indifferent to sending a further message if this holds with equality). Define $X=\frac{\gamma}{\pi\left(\theta_{1}\right)}$, and so this condition is

$$
X(1-X) \leq \frac{\gamma}{\pi(1)}
$$

The LHS is maximized at $X=0.5$. Therefore, all senders will send at most one message if $\gamma \geq \frac{\pi(1)}{4}$ (and there will be some senders if $\gamma<\pi(1)$ ). As noted above, for $k=1$ there can only be one equilibrium for any $\phi$. If $\gamma<\frac{\pi(1)}{4}$ then (8) implies that $\Phi\left(\theta_{1}\right)$ involves $k=2$ for some $\theta_{1}$. Choosing the corresponding value of $\phi$ suffices to sustain such $\theta_{1}$ as an equilibrium. Q.E.D.

Property 3. Assume that $\pi(\theta)=\bar{\pi} \theta$. For any $\theta \in(\gamma / \bar{\pi}, 1]$ the conditional examination functions $\Phi_{i}\left(\theta_{1}\right)$ are quasi-concave.

Proof: Note first that $\Phi_{i}\left(\theta_{1}\right)=\frac{n_{i}\left(\theta_{1}\right) \gamma}{\bar{\pi} \theta_{1}}$. Hence $\frac{\bar{\pi}}{\gamma} \frac{d \Phi_{i}}{d \theta_{1}}=\frac{n_{i}^{\prime}\left(\theta_{1}\right) \theta_{1}-n_{i}\left(\theta_{1}\right)}{\left(\theta_{1}\right)^{2}}$. For the linear formulation,

$$
n_{i}\left(\theta_{1}\right)=i-\theta_{1} \sum_{j=1 \ldots i} \frac{1}{\left(1-\frac{\gamma}{\bar{\pi} \theta_{1}}\right)^{j-1}} .
$$


On the interior of the domain of $\Phi_{i}\left(\theta_{1}\right), n_{i}^{\prime}\left(\theta_{1}\right)=-\sum_{j=1 \ldots i . .} \frac{1-\frac{\gamma}{\bar{\pi} \theta_{1}} j}{\left(1-\frac{\gamma}{\bar{\pi} \theta_{1}}\right)^{j}}$. Hence,

$$
\frac{\bar{\pi}}{\gamma}\left(\theta_{1}\right)^{2} \frac{d \Phi_{i}\left(\theta_{1}\right)}{d \theta_{1}}=-i+\frac{\gamma}{\bar{\pi}} \sum_{j=1 \ldots i} \frac{j-1}{\left(1-\frac{\gamma}{\bar{\pi} \theta_{1}}\right)^{j}}=\Omega_{i} .
$$

This expression implies that $\Phi_{i}\left(\theta_{1}\right)$ is quasi-concave since the factor on the LHS is positive and so $\frac{d \Phi_{i}}{d \theta_{1}}$ has the sign of the RHS. The RHS is decreasing in $\theta_{1}$ : this implies quasi-concavity because $\frac{d \Phi_{i}}{d \theta_{1}}$ is either of the same sign throughout its range so $\Phi_{i}$ is always decreasing (or increasing) or else it switches sign from positive to negative (so that $\Phi_{i}$ is increasing and then decreasing). Q.E.D.

Proof of Proposition 4. As per Lemma 1(ii), an equilibrium with just one message per sender can be achieved through appropriate choice of $\gamma$ if and only if $\left(1-\theta_{1}\right) M \leq \phi$ for the specified $\theta_{1}$ (where we recall that $\left(1-\theta_{1}\right) M$ is the mass of types sending). Such an equilibrium can then be attained by choosing a transmission rate $\hat{\gamma}=\pi\left(\theta_{1}\right)$. This entails an increase in the transmission cost because originally we had $\gamma=\frac{\phi}{n} \pi\left(\theta_{1}\right)$ determining $\theta_{1}$, with $n>\phi$, (i.e., congestion): at such a transmission cost, with one message per sender, the number of messages sent is $\left(1-\theta_{1}\right) M \leq \phi$.

It remains to prove that all senders are no worse off given $\hat{\gamma}$ when $\left(1-\theta_{1}\right) M \leq \phi$. Clearly the marginal sender is indifferent between the original cost $\gamma$ and the higher cost $\hat{\gamma}$ because it makes zero profit before and after. We now show that profits are higher for all senders $\theta>\theta_{1}$; equivalently, we establish that

$$
\pi(\theta)-\hat{\gamma}>\pi(\theta)\left[1-\left(1-\frac{\phi}{n}\right)^{l^{*}}\right]-l^{*} \gamma \quad \text { for all } \theta>\theta_{1},
$$

where $l^{*}$ denotes the number of messages sent by a sender of type $\theta$ at the original equilibrium. The LHS of this expression is the profit per sender at the new equilibrium (when its message is examined for sure, and it sends only one, at cost $\hat{\gamma}$ ); the RHS is its original profit. We also know that, by construction, $\pi\left(\theta_{1}\right)=\hat{\gamma}$ and $\pi\left(\theta_{1}\right) \frac{\phi}{n}=\gamma$, so that $\hat{\gamma}=\frac{n}{\phi} \gamma$. After replacing this expression, we therefore want to show that $\pi(\theta)-\hat{\gamma}>\pi(\theta)\left[1-\left(1-\frac{\phi}{n}\right)^{l^{*}}\right]-l^{*} \frac{\phi}{n} \hat{\gamma}$, or

$$
\pi(\theta)\left(1-\frac{\phi}{n}\right)^{l^{*}}>\left(1-l^{*} \frac{\phi}{n}\right) \hat{\gamma}
$$


which is true for $\theta>\theta_{1}$ since $\pi(\theta)>\pi\left(\theta_{1}\right)=\hat{\gamma}$ because the inequality $\left(1-\frac{\phi}{n}\right)^{l^{*}}>1-l^{*} \frac{\phi}{n}$ holds strictly for $l^{*}>1$. Q.E.D.

\section{References}

[1] Anderson, Chris (2006). The Long Tail: Why the Future of Business Is Selling Less of More. New York: Hyperion.

[2] Anderson, Simon P. And Andre de Palma (2009) Information Congestion. RAND Journal of Economics, 40(4), 688-709.

[3] Anderson, Simon P. And Andre de Palma (2012) Competition for attention in the information (overload) age. RAND Journal of Economics, 43(1), 1-25.

[4] Bagwell, Kyle (2007) The Economic Analysis of Advertising. In Handbook of Industrial Organization, III, (eds. Mark Armstrong and Rob Porter), Elsevier Science, North Holland.

[5] Bergemann, Dirk, And Alessandro Bonatti (2011) Targeting in Advertising Markets: Implications for Offline vs. Online Media. RAND Journal of Economics, 42, 417-433.

[6] Brown, Tom J., and Michael L. Rothschild (1993) Reassessing the Impact of Television Advertising Clutter, Journal of Consumer Research, 20(1), 138-146.

[7] Butters, Gerard R. (1977) Equilibrium Distributions of Sales and Advertising Prices. Review of Economic Studies, 44, 465-491.

[8] Christou, C. and Vettas, Nikos (2008) On informative advertising and product differentiation. International Journal of Industrial Organization, 26(1), 92-112.

[9] De Corniere, Alexandre (2011) Search Advertising. Mimeo, Paris School of Economics.

[10] Eppler, Martin J., and Jeanne Mengis (2004) The Concept of Information Overload: a Review of Literature from Organization Science, Accounting, Marketing, MIS, and Related Disciplines, The Information Society, 20, 325-344. 
[11] Grossman, Gene M., and Shapiro, Carl (1984) Informative Advertising and Differentiated Products. Review of Economic Studies, 51, 63-81.

[12] Hammer, Peter, Erica Riebe, and Rachel Kennedy (2009) How Clutter affects advertising effectiveneness, Journal of Advertising Research, 49(2), 159-163.

[13] Iyer, G., D. Soberman, and J. M. Villas-Boas (2005) The targeting of advertising. Marketing Science, 24(3), 461-476.

[14] Johnson, Justin (2011) Targeted Advertising and Advertising Avoidance. Working Paper, Johnson School, Cornell University.

[15] Kuksov, Dmitri and J. Miguel Villas-Boas (2010) When More Alternatives Lead to Less Choice. Marketing Science, 29 (3), 507-524.

[16] Miller, George A. (1956) The Magical Number Seven, Plus or Minus Two: Some Limits on Our Capacity for Processing Information. The Psychological Review, 63, 81-97.

[17] Pechmann, C., and Stewart, D. W. (1988) Advertising Repetition: A Critical Review of Wearin and Wearout. Current Issues and Research in Advertising, 11, 285-330.

[18] Pieters, Rik, Luk Warlop, and Michel Wedel (2002) Breaking Through the Clutter: Benefits of Advertisement Originality and Familiarity for Brand Attention and Memory. Management Science, 48, 765-781.

[19] Simon, Julian, and Johan Arndt (1980) The shape of the advertising response function. Journal of Advertising Research, 20, 11-28.

[20] Sovinsky Goeree, Michelle (2008) Limited Information and Advertising in the US Personal Computer Industry. Econometrica 76(5), 1017-1074.

[21] Taylor, Jennifer, Rachel Kennedy, and Byron Sharp (2009) Is once really enough? Making generalizations about advertising's convex sales response function. Journal of Advertising Research, 49(2), 198- 200. 
[22] Terui, Nobuhiko, Masataka Ban, and Greg M. Allenby (2011) The Effect of Media Advertising on Brand Consideration and Choice. Marketing Science, 30(1), 74-91.

[23] Van Zandt, Timothy (2004) Information Overload in a Network of Targeted Communication, RAND Journal of Economics, 35(3), 542-560.

[24] Webb, Peter H., And Michael L. Ray (1979) Effects of TV Clutter, Journal of Advertising Research, 19(3), 7-12.

[25] Wind, Yoram (Jerry), and Byron Sharp (2009) Advertising Empirical Generalizations: Implications for Research and Action, Journal of Advertising Research, 49(2), 246-252. 